\title{
Outage Analysis in SWIPT-Based Decode-and-Forward Relay Networks with Partial Relay Selection
}

\author{
Phu Tran Tin $\mathbb{D}^{1},{ }^{1}$ Van-Duc Phan $\mathbb{D}^{2},{ }^{2}$ Phu X. Nguyen $\mathbb{D},{ }^{3}$ Thanh-Long Nguyen $\mathbb{D}^{4}{ }^{4}$ \\ Dong-Si Thien Chau $\mathbb{1}^{5},{ }^{5}$ and Tan N. Nguyen $\mathbb{1}^{6}$ \\ ${ }^{1}$ Faculty of Electronics Technology, Industrial University of Ho Chi Minh City, Ho Chi Minh City, Vietnam \\ ${ }^{2}$ Faculty of Automobile Technology, Van Lang University, Ho Chi Minh City, Vietnam \\ ${ }^{3}$ Department of Computer Fundamentals, FPT University, Ho Chi Minh City, Vietnam \\ ${ }^{4}$ Faculty of Information Technology, Ho Chi Minh City University of Food Industry, Ho Chi Minh City, Vietnam \\ ${ }^{5}$ Modeling Evolutionary Algorithms Simulation and Artificial Intelligence, Faculty of Electrical \& Electronics Engineering, Ton Duc \\ Thang University, Ho Chi Minh City, Vietnam \\ ${ }^{6}$ Wireless Communications Research Group, Faculty of Electrical and Electronics Engineering, Ton Duc Thang University, \\ Ho Chi Minh City, Vietnam
}

Correspondence should be addressed to Thanh-Long Nguyen; longnt@hufi.edu.vn

Received 22 March 2021; Revised 10 May 2021; Accepted 11 June 2021; Published 28 June 2021

Academic Editor: Dimitrios E. Manolakos

Copyright (c) 2021 Phu Tran Tin et al. This is an open access article distributed under the Creative Commons Attribution License, which permits unrestricted use, distribution, and reproduction in any medium, provided the original work is properly cited.

\begin{abstract}
This work studies the SWIPT-based half-duplex (HD) decode-and-forward (DF) relay network, wherein the relay user can scavenge power from the source's radio-frequency (RF) signals and then utilize it to convey the information to the destination. Specifically, two SWIPT-based relaying schemes, termed static power splitting- (SPS-) based relaying (SPSR) and optimal dynamic power splitting- (DPS-) based relaying (ODPSR), are proposed to investigate the benefits of each one fully. Based on the above discussions, the relaying system's performance for outage probability (OP) is studied. Concretely, we derive the analytical expressions for both SPSR and DPSR methods. Finally, the numerical simulations are executed to corroborate the analysis and simulation results.
\end{abstract}

\section{Introduction}

With the unprecedented growth of wireless data traffic and IoT devices (IoTDs), energy consumption in wireless communications has increased significantly in the last few decades [1-4]. As reported in [5-7], the number of IoT users is estimated to reach 11.6 billion and 25 billion by 2021 and 2025, respectively. Nevertheless, IoTDs often have battery capacity limitations, which restrict the total operation time. Moreover, battery recharging and/or replacement can be expensive and even impossible such as inside human bodies or toxic environments. Fortunately, energy harvesting (EH) has emerged as a promising technique to overcome these issues [8]. In particular, radio frequency (RF) $\mathrm{EH}$ has received significant attention from both industry and academia recently because it does not depend on natural weather, i.e., solar [9], wind [10], and water [8], and is controllable [11-27]. Notably, RF signals can bring both information and energy to the receiver simultaneously, termed SWIPT. Varshney is a pioneer of the SWIPT concept [23]. Then, Zhang and Ho [13] proposed practical system models for SWIPT, namely, time-switching (TS) and power-splitting (PS) methods. Based on TS and PS in [13], Nasir et al. [16] proposed two relaying protocols, termed time switchingbased relaying (TSR) protocol and power splitting-based relaying (PSR) protocol, to introduce $\mathrm{EH}$ and data transmission at the relay node in cooperative wireless networks. In $[24,25]$, the authors investigated the dynamic PS-based SWIPT with dual-hop decode-and-forward (DF) relaying in the presence of a direct link. Specifically, they derived the exact closed-form expressions for outage probability (OP) and ergodic capacity (EC). Besides, they derived the optimal 
value of PS to minimize the OP at a given threshold rate. Shi et al. [26] studied a SWIPT-based three-step two-way DF relay network with a nonlinear energy harvester equipped at a relay. Specifically, they derived the closed-form expressions for OP and network capacity, where the PS ratio is dynamically changed according to the instantaneous channel state information (CSI). Ye et al. [27] designed optimal static and dynamic transmission schemes in SWIPT-based DF relay networks. Concretely, they obtained the optimal values of time allocation (TA) and PS ratios via solving two optimization problems, namely, outage probability minimization and instantaneous channel capacity maximization.

This work analyzed the performance of the SWIPT-based half-duplex (HD) DF relay system, whereas the relay user scavenges power from the source RF signals and then uses it to transmit data to the destination. This research's contributions are given as follows:

(i) We propose a novel system model of a SWIPTenabled DF relaying network with the PSR protocol. Moreover, we propose two SWIPT-based relaying schemes, namely, SPS-based relaying (SPSR) and optimal DPS-based relaying (ODPSR), to study each one's advantages fully

(ii) Based on the proposed system model, we derive the analysis expressions of outage probability for SPSR and ODPSR schemes

(iii) The Monte Carlo simulations are presented to corroborate the mathematic analysis. Specifically, we also present an insightful analysis of the effectiveness of different system parameters on the system performance, i.e., source transmit power, number of relay nodes, energy harvesting coefficient, and rate threshold

The remainder of this paper is structured. The system model of the SWIPT-assisted DF relay system is presented in Section 2. Then, the OP analysis is given in Section 3. Simulation results are described in Section 4.

\section{System Model}

2.1. Energy Harvesting and Transmission Model. We consider a SWIPT-assisted DF relaying network as in Figure 1, with one source $S$; multiple relays, denoted by $R_{n}$ with $n \in\{1,2$, $\cdots, N\}$; and one destination $D$. Furthermore, the direct transmission link from $S \longrightarrow D$ is missing due to heavy obstacles. Further, $S$, relays, and $D$ are equipped with one antenna and operate on the HD mode. Notably, the relay node can harvest energy from the source's RF signals. Then, the selected relay uses the harvested energy for relaying the source's data to the destination. As shown in Figure 2, the total operation time $T$ is divided into two equal parts, i.e., $T / 2$. In the first half of the time, the relay node harvests energy from a part of the source's signals, i.e., $\rho P_{S}$, using the power-splitting method, where $0 \leq \rho \leq 1$ denotes the power-switching factor. The remained power, i.e., $(1-\rho) \mathrm{P}_{\mathrm{S}}$, is used for information decoding. In the remaining half of the time, the selected relay

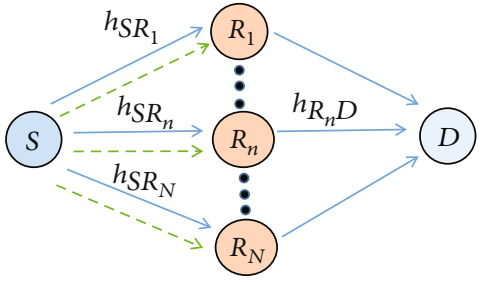

$\rightarrow$ Energy flow

$\longrightarrow$ Information flow

Figure 1: System model.

\begin{tabular}{|c|c|}
\hline \multicolumn{2}{|c|}{$T$} \\
\hline $\begin{array}{c}\text { Energy harvesting at } R_{\mathrm{n}} \\
\rho P_{S}\end{array}$ & Information transmission \\
\hline $\begin{array}{c}\text { Information transmission } \\
(1-\rho) P_{S}\end{array}$ & $R_{n} \longrightarrow D$ \\
\hline$T / 2$ & \\
\hline
\end{tabular}

Figure 2: IT and EH processes with power-splitting relaying protocols.

uses all harvested energy for relaying information to the destination $D$. Further, we assume that the channel between two arbitrary nodes is a block Rayleigh fading.

Let us denote $h_{S R_{n}}$ and $h_{R_{n} D}$ as the channel coefficients of the $S \longrightarrow R_{n}$ and $R_{n} \longrightarrow D$ links, respectively.

Assume that all of the channels are Rayleigh fading. Hence, the channel gains $\gamma_{S R_{n}}=\left|h_{S R_{n}}\right|^{2}$ and $\gamma_{R_{n} D}=\left|h_{R_{n} D}\right|^{2}$ are exponential random variables (RVs) whose CDF are given as

$$
\begin{gathered}
F_{\gamma_{S R_{n}}}(x)=1-\exp \left(-\lambda_{S R_{n}} x\right), \\
F_{\gamma_{R_{n} D}}(x)=1-\exp \left(-\lambda_{R_{n} D} x\right) .
\end{gathered}
$$

To take the path-loss model into account, we have

$$
\lambda_{S R_{n}}=\left(d_{S R_{n}}\right)^{\beta}, \lambda_{R_{n} D}=\left(d_{R_{n} D}\right)^{\beta}
$$

where $d_{S R_{n}}$ and $d_{R_{n} D}$ are link distances of the $S \longrightarrow R_{n}$ and $R_{n} \longrightarrow D$ links, respectively.

Then, the PDFs of $\gamma_{S R_{n}}, \gamma_{R_{n} D}$ are given, respectively, as

$$
\begin{gathered}
f_{\gamma_{S R_{m}}}(x)=\lambda_{S R_{m}} \exp \left(-\lambda_{S R_{m}} x\right), \\
f_{\gamma_{R_{m} D}}(x)=\lambda_{R_{m} D} \exp \left(-\lambda_{R_{m} D} x\right) .
\end{gathered}
$$

The received signal at the relay $n$-th can be expressed as

$$
y_{R_{n}}=\sqrt{1-\rho} h_{S R_{n}} x_{S}+n_{R_{n}},
$$


where $x_{S}$ is the energy symbol with $E\left\{\left|x_{S}\right|^{2}\right\}=P_{S}$ which $E\{\cdot\}$ denotes the expectation operation. $n_{R_{n}}$ is the zero mean additive white Gaussian noise (AWGN) with variance $N_{0}$.

The transmit power at relay $R_{n}$ can be computed as [12]

$$
P_{R_{n}}=\frac{E_{n}}{T / 2}=\eta \rho P_{S} \gamma_{S R_{n}}
$$

where $P_{S}$ is the transmit power at the source $S$.

The received signal at the destination can be given as

$$
y_{D}=h_{R_{n} D} x_{R_{n}}+n_{D}
$$

where $n_{D}$ is the zero mean AWGN with variance $N_{0}$.

From (4), the signal-to-noise ratio (SNR) at the relay $n$-th node can be derived by

$$
\gamma_{R_{n}}=\frac{(1-\rho) \gamma_{S R_{n}} P_{S}}{N_{0}}=(1-\rho) \gamma_{S R_{n}} \Phi
$$

where $\Phi=P_{S} / N_{0}$.

From (6), the SNR at the destination can be obtained as

$$
\gamma_{D}=\frac{P_{R_{n}} \gamma_{R_{n} D}}{N_{0}}=\eta \rho \Phi \gamma_{S R_{n}} \gamma_{R_{n} D}
$$

Finally, the overall SNR and capacity of the system and can be, respectively, given by

$$
\begin{aligned}
& \psi_{\mathrm{DF}}=\min \left(\gamma_{R_{n}}, \gamma_{D}\right), \\
& C_{\mathrm{DF}}=\frac{1}{2} \log _{2}\left(1+\psi_{\mathrm{DF}}\right) .
\end{aligned}
$$

2.2. Partial Relay Selection (PRS). In this paper, we apply the partial relay selection (PRS) method to improve communication performance. Specifically, the best relay can be selected among $N$ relay nodes as follows:

$$
R_{a}: \gamma_{S R_{a}}=\max _{n=1,2, \cdots, N}\left(\gamma_{S R_{n}}\right)
$$

From (10), the relay with the best channel from the source node to itself is selected as the best relay.

The CDF $F_{\gamma_{\max }}(x)$ can be given by

$$
F_{\gamma_{S R_{a}}}(x)=\operatorname{Pr}\left(\gamma_{S R_{a}}<x\right)=\prod_{m=1}^{M} F_{\gamma_{S R_{m}}}(x) .
$$

By considering the independent and identical distributed (i.i.d.) random variables (RVs), i.e., $\lambda_{S R_{n}}=\lambda_{S R}, \forall n$, equation (11) can be rewritten as

$$
\begin{aligned}
F_{\gamma_{S R_{a}}}(x) & =\left[1-\exp \left(-\lambda_{S R} x\right)\right]^{N} \\
& =1+\sum_{k=1}^{N}(-1)^{k} C_{N}^{k} \exp \left(-k \lambda_{S R} x\right),
\end{aligned}
$$

where $C_{N}^{k}=N ! / k !(N-k) !$

\section{Outage Probability (OP) Analysis}

3.1. Case 1: Static Power Splitting-Based Relaying. The OP at the destination can be defined as

$$
\mathrm{OP}=\operatorname{Pr}\left(C_{\mathrm{DF}}<C_{\mathrm{th}}\right)=\operatorname{Pr}\left(\psi_{\mathrm{DF}}<\gamma_{\mathrm{th}}\right)
$$

where $\gamma_{\mathrm{th}}=2^{2 C_{\mathrm{th}}}-1$, and $C_{\mathrm{th}}$ is the deterministic threshold rate.

By combining with (7) and (8), (13) can be rewritten as

$$
\begin{aligned}
\mathrm{OP}= & \operatorname{Pr}\left(\min \left((1-\rho) \gamma_{S R_{a}} \Phi, \eta \rho \Phi \gamma_{S R_{a}} \gamma_{R_{a} D}\right)<\gamma_{\mathrm{th}}\right) \\
= & 1-\operatorname{Pr}\left((1-\rho) \gamma_{S R_{a}} \Phi \geq \gamma_{\mathrm{th}}, \eta \rho \Phi \gamma_{S R_{a}} \gamma_{R_{a} D} \geq \gamma_{\mathrm{th}}\right) \\
= & 1-\operatorname{Pr}\left(\gamma_{S R_{a}} \geq \frac{\gamma_{\mathrm{th}}}{(1-\rho) \Phi}, \gamma_{S R_{a}} \gamma_{R_{a} D} \geq \frac{\gamma_{\mathrm{th}}}{\eta \rho \Phi}\right) \\
= & 1-\int_{0}^{\xi} f_{\gamma_{R_{a} D}}(y) d y \int_{\gamma_{\mathrm{th}} / \eta \rho \Phi y}^{\infty} f_{\gamma_{S R_{a}}}(x) d x \\
& \quad-\int_{\xi}^{\infty} f_{\gamma_{R_{a} D}}(y) d y \int_{\vartheta}^{\infty} f_{\gamma_{S R_{a}}}(x) d x,
\end{aligned}
$$

where $\vartheta=\gamma_{\text {th }} /(1-\rho) \Phi, \xi=(1-\rho) / \eta \rho$.

By applying (12), (14) can be obtained as

$$
\begin{aligned}
\mathrm{OP}= & 1+\sum_{k=1}^{N}(-1)^{k} C_{N}^{k} \exp \left(-\lambda_{R_{a} D} \xi-k \lambda_{S R} \vartheta\right) \\
& +\sum_{k=1}^{N}(-1)^{k} C_{N}^{k} \lambda_{R_{a} D} \int_{0}^{\xi} \exp \left(-\frac{k \lambda_{S R} \gamma_{\mathrm{th}}}{\eta \rho \Phi y}-\lambda_{R_{a} D} y\right) d y
\end{aligned}
$$

3.2. Case 2: Optimal Dynamic Power Splitting-Based Relaying. From (9), in order to enhance the system quality, we will try to find the optimal $\rho^{*}$ value to maximize $C_{\mathrm{DF}}$. Because we consider the DF protocol in our model, the $\rho^{*}$ can be calculated by solving the following equation [28]:

$$
\gamma_{R_{n}}=\gamma_{D} \leftrightarrow(1-\rho) \gamma_{S R_{n}} \Phi=\eta \rho \Phi \gamma_{S R_{n}} \gamma_{R_{n} D} \longrightarrow \rho^{*}=\frac{1}{\eta \gamma_{R_{n} D}+1}
$$

Substituting (16) into (7), the OP in this case can be expressed by 


$$
\begin{aligned}
\mathrm{OP}^{*} & =\operatorname{Pr}\left(\frac{\eta \Phi \gamma_{S R_{a}} \gamma_{R_{a} D}}{\eta \gamma_{R_{a} D}+1}<\gamma_{\mathrm{th}}\right) \\
& =\operatorname{Pr}\left(\gamma_{S R_{a}}<\frac{\gamma_{\mathrm{th}}\left(\eta \gamma_{R_{a} D}+1\right)}{\eta \Phi \gamma_{R_{a} D}}\right) \\
& =\int_{0}^{\infty} F_{\gamma_{R_{a}}}\left(\frac{\gamma_{\mathrm{th}}(\eta x+1)}{\eta \Phi x}\right) f_{\gamma_{R_{a} D}}(x) d x .
\end{aligned}
$$

By applying (12), (17) can be reformulated as

$$
\begin{aligned}
\mathrm{OP}^{*}= & 1+\sum_{k=1}^{N}(-1)^{k} C_{N}^{k} \int_{0}^{\infty} \lambda_{R_{a} D} \exp \left(-\frac{k \lambda_{S R} \gamma_{\mathrm{th}}(\eta x+1)}{\eta \Phi x}-\lambda_{R_{a} D} x\right) d x \\
= & 1+\sum_{k=1}^{N}(-1)^{k} C_{N}^{k} \exp \left(-\frac{k \lambda_{S R} \gamma_{\mathrm{th}}}{\Phi}\right) \int_{0}^{\infty} \lambda_{R_{a} D} \exp \\
& \cdot\left(-\frac{k \lambda_{S R} \gamma_{\mathrm{th}}}{\eta \Phi x}-\lambda_{R_{a} D} x\right) d x .
\end{aligned}
$$

Finally, with the help of ([29], 3.324.1), OP* can be claimed by

$$
\begin{aligned}
\mathrm{OP}^{*}= & +2 \sum_{k=1}^{N}(-1)^{k} C_{N}^{k} \exp \left(-\frac{k \lambda_{S R} \gamma_{\mathrm{th}}}{\Phi}\right) \sqrt{\frac{k \lambda_{S R} \lambda_{R_{a} D} \gamma_{\mathrm{th}}}{\eta \Phi}} \\
& \times K_{1}\left(2 \sqrt{\frac{k \lambda_{S R} \lambda_{R_{a} D} \gamma_{\mathrm{th}}}{\eta \Phi}}\right),
\end{aligned}
$$

where $K_{v}(\cdot)$ is the modified Bessel function of the second kind and $v$-th order.

\section{Simulation Results}

This part presents the numerical results to show the impacts of various parameters on the outage performance for the proposed SWIPT-enabled DF relaying network with PSR using Monte Carlo simulations [28, 30-34]. Without loss of generality, we assume that the distances between $S \longrightarrow R_{n}$ and $R_{n} \longrightarrow D$ are equal to the unit value. The mean values of channel gain coefficients $\lambda_{S R}$ and $\lambda_{R D}$, respectively, equal to 0.5 and $1 ; \phi$ value varies from -5 to $15 \mathrm{~dB}$; and the energy harvesting coefficient is $\eta=0.8$. The simulation results for outage probability are obtained by averaging it over $10^{6} \mathrm{sam}-$ ples for each Rayleigh fading channel.

Figure 3 depicts the outage of the proposed system for varying $\phi(\mathrm{dB})$. It can be seen that the performance of the optimal DPS-based relaying (ODPSR) is better than that of SPS-based relaying (SPSR) with $\rho$ equals 0.25 and 0.75 . Further, when the value of $\phi$ is less than $6 \mathrm{~dB}$, the OP of the SPSR with $\rho=0.25$ is better than that of the SPSR with $\rho=0.75$. Nevertheless, when the value of $\phi$ is higher than $6 \mathrm{~dB}$, the outage performance of the SPSR with $\rho=0.25$ is worse than that of the SPSR with $\rho=0.75$. Further, the increasing of the $\phi$ value significantly influences the OP of all schemes. It is

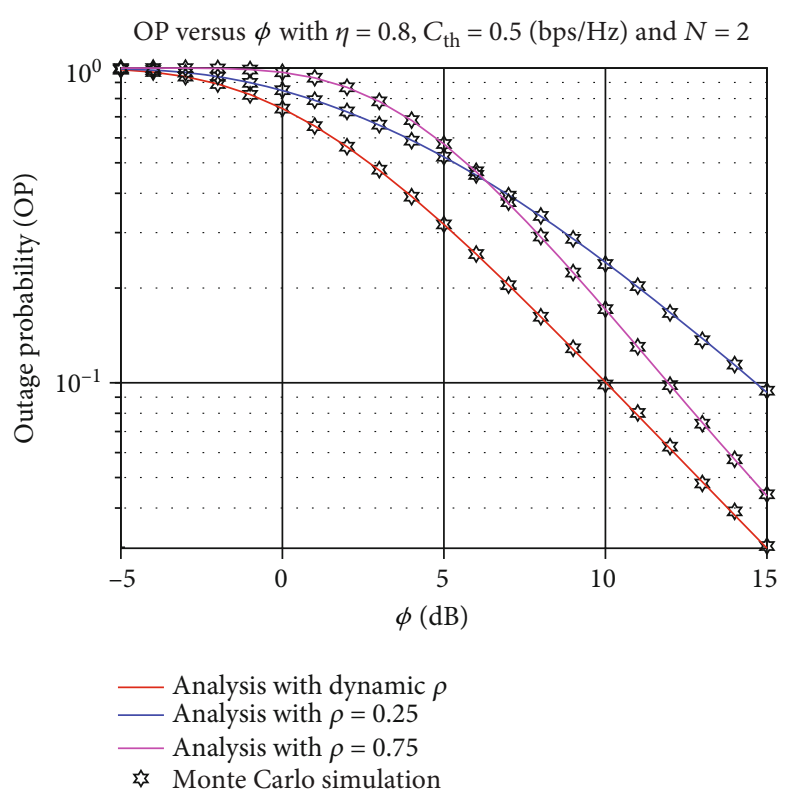

Figure 3: Outage probability versus $\Psi$.

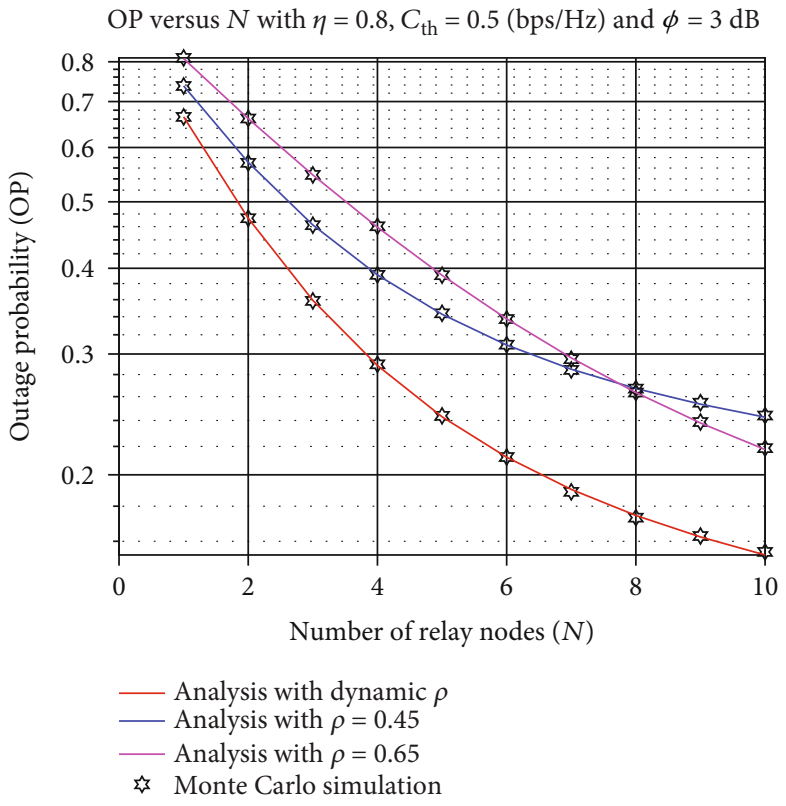

FIGURE 4: Outage probability versus a number of sources $(N)$.

expected because the value of $\phi$ is defined as the ratio between the source's transmit power and noise power. Therefore, the higher the $\phi$ value is, the more the source's transmit power. As a result, the destination can obtain a larger data rate, which reduces the outage value.

In Figure 4, we study the outage probability as a function of the number of relay nodes $(N)$. As expected, the performance of the ODPSR method obtains the best results as compared with that of the SPS methods, i.e., SPSR with $\rho$ equals 0.45 and 0.65 . This is due to the fact that the ODPSR scheme is aimed at finding the optimal value of $\rho$ to maximize the received capacity at the destination. Another interesting 


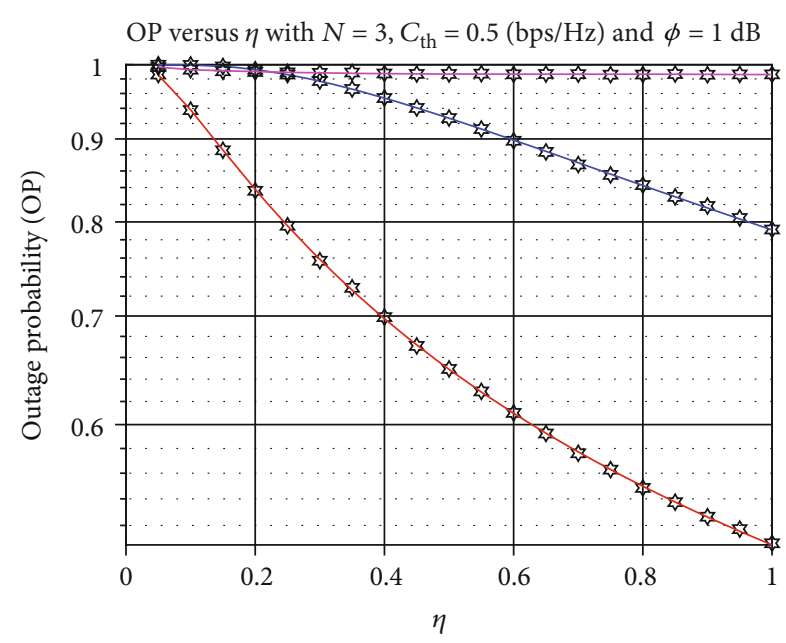

— Analysis with dynamic $\rho$

— Analysis with $\rho=0.15$

— Analysis with $\rho=0.85$

\& Monte Carlo simulation

FIGURE 5: Outage probability versus $\eta$.

OP versus $C_{\text {th }}$ with $N=3, \eta=0.8$ and $\phi=3 \mathrm{~dB}$

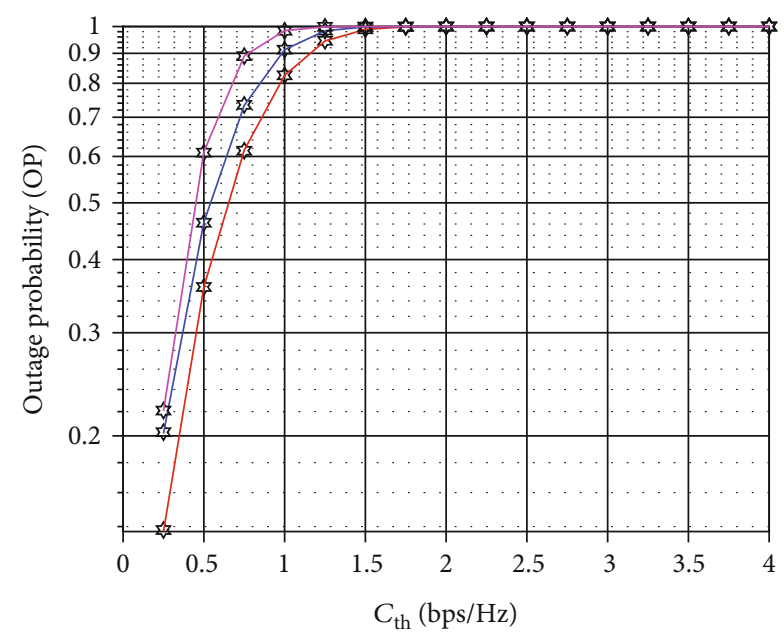

_ Analysis with dynamic $\rho$

- Analysis with $\rho=0.5$

_ Analysis with $\rho=0.7$

is Monte Carlo simulation

FIgure 6: Outage probability versus $C_{\text {th }}(\mathrm{bps} / \mathrm{Hz})$.

point is that when the number of relays is less than 8 , the SPSR with $\rho$ equal to 0.45 achieves a lower outage value than the SPSR with $\rho$ equal to 0.65 . Otherwise, when the number of relays is higher than or equals 8 , the SPSR with $\rho$ equal to 0.45 attains a higher OP value than the SPSR with $\rho$ equal to 0.65 .

Figure 5 illustrates the OP of ODPSR and SPSR versus energy harvesting efficiency $\eta$, with $N=3, \phi=1 \mathrm{~dB}$, and $C_{\text {th }}$ $=0.5 \mathrm{bps} / \mathrm{Hz}$. From Figure 5 , we see that there is good agreement between the mathematical analysis and numerical results. As is shown in this figure, when $\eta$ increases, the OP decreases because the harvested energy at the relay is propor- tional to the energy harvesting efficiency. Moreover, this can be interpreted by its expression in equations (5) and (8). Specifically, the higher the $\eta$ value is, the larger the destination's data rate that can be obtained. Thus, the outage performance can be improved.

Figure 6 shows the OP as a function of $C_{\mathrm{th}}$, with $N=3$, $\phi=3 \mathrm{~dB}$, and $\eta=0.8$. As shown in Figure 6 , the value of $C_{\mathrm{th}}$ significantly influences the outage performance. Specifically, the higher the value of $C_{t h}$ is, the worse is the performance obtained. This can be explained that the higher the value of $C_{\text {th }}$ is, the larger the transmission rate that is needed to decode the received signals at the destination successfully. However, the received rate is unchanged due to the limited transmit power at the source. Further, it is also observed that the OP value converges to a saturation value when the $C_{\text {th }}$ is large enough, i.e., $C_{\text {th }} \geq 1.5 \mathrm{bps} / \mathrm{Hz}$.

\section{Conclusions and Future Directions}

This work proposed a new PSR protocol for a SWIPTenabled relaying network over DF-based Rayleigh fading channels. The system model included one source, multiple relays, and one destination for the data transmission from source $S$ to destination $D$. To find the best relay node, we proposed one partial relay selection protocol. Based on the proposed system model, we derive the analytical expressions of outage probability for both SPSR and ODPSR schemes. Then, we investigated the influence of all designed parameters on the system performance by using a Monte Carlo simulation. Numerical results showed that the ODPSR was better compared with SPSR schemes. This work can be extended in future work by considering satellite communications, hardware impairment, or NOMA. Another promising direction is to consider the nonlinear energy harvesting model or jointly consider time allocation and the PS ratio that can boost the performance for the DF relaying network, which requires many challenges.

\section{Data Availability}

There is no available data in our work.

\section{Conflicts of Interest}

The authors declare that they have no conflicts of interest.

\section{Authors' Contributions}

The main contribution of Phu Tran Tin (phutrantin@iuh.edu.vn) was to execute performance evaluations by theoretical analysis and simulations, while Phan Van-Duc (duc.pv@vlu.edu.vn.), Phu X. Nguyen (phunx4@fpt.edu.vn, phunx4@fe.edu.vn), and Tan N. Nguyen (nguyennhattan@tdtu.edu.vn) worked as the supervisors of Phu Tran Tin. Thanh-Long Nguyen and Dong-Si Thien Chau (dongsithienchau@tdtu.edu.vn) helped us to improve the manuscript in a revised version. 


\section{Acknowledgments}

This research was supported by the Industrial University of Ho Chi Minh City (IUH), Vietnam, under grant No. 72/HD-DHCN.

\section{References}

[1] W. Lu, P. Si, G. Huang et al., "SWIPT cooperative spectrum sharing for $6 \mathrm{G}$-enabled cognitive IoT network," IEEE Internet of Things Journal, 2020.

[2] P. X. Nguyen, D.-H. Tran, O. Onireti et al., "Backscatterassisted data offloading in OFDMA-based wireless powered mobile edge computing for IoT networks," IEEE Internet of Things Journal, vol. 8, no. 11, pp. 9233-9243, 2021.

[3] Y. Hu, Y. Zhu, M. C. Gursoy, and A. Schmeink, "SWIPTenabled relaying in IoT networks operating with finite blocklength codes," IEEE Journal on Selected Areas in Communications, vol. 37, no. 1, pp. 74-88, 2019.

[4] Z. Hasan, H. Boostanimehr, and V. K. Bhargava, "Green cellular networks: a survey, some research issues and challenges," IEEE Communications Surveys \& Tutorials, vol. 13, no. 4, pp. 524-540, 2011.

[5] Cisco, Cisco visual networking index: global mobile data traffic forecast update, 2016-2021, white paper, Cisco, San Jose, CA, USA, 2017.

[6] D.-H. Tran, V.-D. Nguyen, S. Gautam, S. Chatzinotas, T. X. $\mathrm{Vu}$, and B. Ottersten, "Resource allocation for UAV relayassisted IoT communication networks," in 2020 IEEE Globecom Workshops (GC Workshops), pp. 1-7, Taipei, Taiwan, 2020.

[7] Ericsson, Ericsson mobility report: November 2019, Ericsson, Stockholm, Sweden, 2019.

[8] D. Fooladivanda, A. D. Domínguez-García, and P. W. Sauer, "Utilization of water supply networks for harvesting renewable energy," IEEE Transactions on Control of Network Systems, vol. 6, no. 2, pp. 763-774, 2019.

[9] T. Hieu, L. Dung, and B. S. Kim, "Stability-aware geographic routing in energy harvesting wireless sensor networks," Sensors, vol. 16 , no. 5 , p. $696,2016$.

[10] L. Zhao, L. Tang, J. Liang, and Y. Yang, "Synergy of wind energy harvesting and synchronized switch harvesting interface circuit," IEEE/ASME Transactions on Mechatronics, vol. 22, no. 2, pp. 1093-1103, 2017.

[11] T. N. Nguyen, P. T. Tran, and M. Voznak, "Wireless energy harvesting meets receiver diversity: a successful approach for two-way half-duplex relay networks over block Rayleigh fading channel," Computer Networks, vol. 172, p. 107176, 2020.

[12] D. H. Ha, C. Dong, T. N. Nguyen, T. T. Trang, and M. Voznak, "Half-duplex energy harvesting relay network over different fading environment: system performance with effect of hardware impairment," Applied Sciences, vol. 9, no. 11, p. 2283, 2019.

[13] R. Zhang and C. K. Ho, "MIMO broadcasting for simultaneous wireless information and power transfer," IEEE Transactions on Wireless Communications, vol. 12, no. 5, pp. 1989-2001, 2013.

[14] T. D. Hieu, T. T. Duy, and B. S. Kim, "Performance enhancement for multi-hop harvest-to-transmit WSNs with pathselection methods in presence of eavesdroppers and hardware noises," IEEE Sensors Journal, vol. 18, no. 12, pp. 5173-5186, 2018.
[15] P. T. Tin, B. H. Dinh, T. N. Nguyen, D. H. Ha, and T. T. Trang, "Power beacon-assisted energy harvesting wireless physical layer cooperative relaying networks: performance analysis," Symmetry, vol. 12, no. 1, p. 106, 2020.

[16] A. A. Nasir, X. Zhou, S. Durrani, and R. A. Kennedy, "Relaying protocols for wireless energy harvesting and information processing," IEEE Transactions on Wireless Communications, vol. 12, no. 7, pp. 3622-3636, 2013.

[17] T. Hieu, T. Duy, L. Dung, and S. Choi, "Performance evaluation of relay selection schemes in beacon-assisted dual-hop cognitive radio wireless sensor networks under impact of hardware noises," Sensors, vol. 18, no. 6, p. 1843, 2018.

[18] S. Atapattu and J. Evans, "Optimal energy harvesting protocols for wireless relay networks," IEEE Transactions on Wireless Communications, vol. 15, no. 8, pp. 5789-5803, 2016.

[19] P. T. Tin, P. Van-Duc, T. N. Nguyen, and L. A. Vu, "Performance analysis for exact and upper bound capacity in DF energy harvesting full-duplex with hybrid TPSR protocol," Journal of Electrical and Computer Engineering, vol. 2021, Article ID 6610107, 9 pages, 2021.

[20] T. N. Nguyen, T. Minh, P. T. Tran, and M. Voznak, “Adaptive energy harvesting relaying protocol for two-way half-duplex system network over Rician fading channels," Wireless Communications and Mobile Computing, vol. 2018, Article ID 7693016, 10 pages, 2018.

[21] P. T. Tin, T. N. Nguyen, M. Tran, T. T. Trang, and L. Sevcik, "Exploiting direct link in two-way half-duplex sensor network over block Rayleigh fading channel: upper bound ergodic capacity and exact SER analysis," Sensors, vol. 20, no. 4, p. 1165, 2020.

[22] D. H. Ha, T. N. Nguyen, M. H. Q. Tran, X. Li, P. T. Tran, and M. Voznak, "Security and reliability analysis of a two-way halfduplex wireless relaying network using partial relay selection and hybrid TPSR energy harvesting at relay nodes," IEEE Access, vol. 8, pp. 187165-187181, 2020.

[23] L. R. Varshney, “Transporting information and energy simultaneously," in 2008 IEEE International Symposium on Information Theory, pp. 1612-1616, Toronto, ON, Canada, 2008.

[24] Y. Ye, Y. Li, F. Zhou, N. Al-Dhahir, and H. Zhang, "Power splitting-based SWIPT with dual-hop DF relaying in the presence of a direct link," IEEE Systems Journal, vol. 13, no. 2, pp. 1316-1319, 2019.

[25] Y. Liu, Y. Ye, and H. Ding, "Dynamic power splitting scheme for DF relaying-based SWIPT networks with direct link," Electronics Letters, vol. 55, no. 25, pp. 1340-1343, 2019.

[26] L. Shi, W. Cheng, Y. Ye, H. Zhang, and R. Q. Hu, "Heterogeneous power-splitting based two-way DF relaying with nonlinear energy harvesting," in 2018 IEEE Global Communications Conference (GLOBECOM), pp. 1-7, Abu Dhabi, United Arab Emirates, 2018.

[27] Y. Ye, Y. Li, D. Wang, F. Zhou, R. Q. Hu, and H. Zhang, "Optimal transmission schemes for DF relaying networks using SWIPT," IEEE Transactions on Vehicular Technology, vol. 67, no. 8, pp. 7062-7072, 2018.

[28] D. Le, D. H. Tran, S. Choi, B. Kim, and B. An, "Impact of beamforming on the path connectivity in cognitive radio adhoc networks," Sensors, vol. 17, 2017.

[29] A. Jeffrey and D. Zwillinger, Table of Integrals, Series, and Products, Elsevier, Amsterdam, Netherlands, 2015.

[30] T. N. Nguyen, T. H. Quang Minh, P. T. Tran et al., "Performance enhancement for energy harvesting based two-way relay protocols in wireless ad-hoc networks with partial and 
full relay selection methods," Ad Hoc Networks, vol. 84, pp. 178-187, 2019.

[31] H. Dinh Tran, D. Trung Tran, and S. G. Choi, "Secrecy performance of a generalized partial relay selection protocol in underlay cognitive networks," International Journal of Communication Systems, vol. 31, no. 17, 2018.

[32] D. H. Ha, T. N. Nguyen, D. V. Phan, T. Phu, M. Tran, and M. Voznak, "Non-linear energy harvesting based power splitting relaying in full-duplex AF and DF relaying networks: system performance analysis," Proceedings of the Estonian Academy of Sciences, vol. 69, no. 4, pp. 368-381, 2020.

[33] T. D. Hieu, T. T. Duy, and S. G. Choi, "Performance enhancement for harvest-to-transmit cognitive multi-hop networks with best path selection method under presence of eavesdropper," in 2018 20th International Conference on Advanced Communication Technology (ICACT), pp. 1-2, Chuncheon, Korea (South), 2018.

[34] M. Ashraf, J. Jang, J. Han, and K. G. Lee, "Capacity maximizing adaptive power splitting protocol for cooperative energy harvesting communication systems," IEEE Communications Letters, vol. 22, no. 5, pp. 902-905, 2018. 